\title{
Women's Personal and Life-Style Characteristics as Related to Molecular Genetic Changes within Their Uterine Cancers
}

\author{
Peter Zauber, $M D^{1^{*}}$, Thad R Denehy, $M D^{2}$, Robert R Taylor, MD², Stephen Marotta, PhD', Marlene \\ Sabbath Solitare, PhD ${ }^{3}$ and Patrick Hilden, $\mathrm{MS}^{4}$
}

${ }^{1}$ Department of Medicine, Saint Barnabas Medical Center, USA

${ }^{2}$ Department of Obstetrics and Gynecology, Saint Barnabas Medical Center, USA

${ }^{3}$ Department of Pathology, Saint Barnabas Medical Center, USA

${ }^{4}$ Department of Biostatistics, Saint Barnabas Medical Center, USA

*Corresponding author: Peter Zauber, MD, 357 Redmond Road, South Orange, NJ 07079, USA, Tel: 973-761-7025

\begin{abstract}
Background: The histopathology and molecular genetics of endometrial cancer have been extensively studied, and they continue to receive attention and new interpretations. However, few studies have evaluated patients' personal and clinical characteristics, or family history, with respect to the tumors' molecular changes. The aim of our study was to evaluate the personal and familial information of patients with respect to several molecular genetic findings of their endometrial cancers.
\end{abstract}

Methods: 105 uterine carcinomas were evaluated for mutations in the genes Ki-Ras, BRAF, GNAS, and PTEN. Loss of heterozygosity of $A P C$ and $P T E N$ was assessed. Tumors were evaluated for microsatellite instability, and methylation was assessed in those tumors with microsatellite instability. An extensive history was obtained from the patients including family history, medical history, and personal characteristics of cigarette, coffee, and medication use.

Results: Age at diagnosis was significantly related to the presence of methylation. BMI under 30 was significantly associated with PTEN mutation. We did not find any significant correlation between the presence of diabetes, hypertension, coffee consumption or use of nonsteroidal anti-inflammatory drugs or cigarettes with the various genetic changes. Estrogen use was associated with microsatellite instability. Vitamin use was more frequent among women with tumors demonstrating PTEN LOH. No correlation was found between any of the genetic changes and whether the patient had a first-degree or second degree relative with uterine cancer.

Conclusions: Although certain germ line genetic changes, as well as unopposed estrogenic effect and obesity may predispose a woman to the development of uterine cancer, the specific molecular changes within the tumor may develop from multiple other exogenous influences, most of which remain undefined. The challenge for the future is to combine large clinical cohorts that have detailed clinical patient information with molecular information of their uterine cancers in an effort to define these influences.

\section{Keywords}

Uterine cancer, Molecular genetic changes, Ki-ras gene mutations, Microsatellite instability, PTEN gene mutations

\section{Abbreviations \\ EC: Endometrial Carcinoma; NHPCC: Hereditary Non-Pol- yposis Colorectal Cancer; MSI: Microsatellite Instability; NSAID: Non-Steroidal Anti-Inflammatory Drug; DES: Dieth- ylstilbesterol; BMI: Body Mass Index}

\section{Introduction}

Endometrial cancer (EC) is the most common gynecological cancer and the fourth most common cancer in women. There are two main subtypes, based on histopathological characteristics. Type I is endometrioid endometrial carcinoma, and represents approximately $80 \%$ of all endometrial cancers. Numerous molecular genetic changes have been identified in endometrial cancers, including mutations in the genes KRAS, BRAF, PTEN and PIK3CA, APC loss of heterozygosity, microsatellite instability, and epigenetic methylation. Type II is non-endometrioid endometrial carcinoma. It is more

Citation: Zauber P, Denehy TR, Taylor RR, Marotta S, Solitare MS, et al. (2020) Women's Personal and Life-Style Characteristics as Related to Molecular Genetic Changes within Their Uterine Cancers. Int J Cancer Clin Res 7:137. doi.org/10.23937/2378-3419/1410137

Accepted: May 20, 2020; Published: May 22, 2020

Copyright: (C) 2020 Zauber P, et al. This is an open-access article distributed under the terms of the Creative Commons Attribution License, which permits unrestricted use, distribution, and reproduction in any medium, provided the original author and source are credited. 
common in post-menopausal women, and it is not usually associated with hyper-estrogenic factors [1,2]. Recently, a new framework was proposed, based primarily upon genomic information [3].

There have been numerous publications on the histopathology and molecular genetics of endometrial cancer $[4,5]$. However, there are very few publications that have looked closely for a relationship between family history and molecular changes, save for the relationship of microsatellite instability (MSI) and the familial syndrome hereditary non-polyposis colorectal cancer (HNPCC) [6]. Further, there are very few publications examining the relationship between personal life-style characteristics (use of drugs, cigarettes, body mass index, etc.) and molecular genetic changes. For example, is cigarette use associated with MSI in uterine cancer, as it is in colorectal cancer? [7]. The aim of our study was to evaluate the personal and family histories of patients with endometrial cancer with respect to several molecular genetic changes within their uterine cancers. Many of these life-style features can be modified, and it would be important to identify any that may be related to somatic molecular changes.

\section{Materials and Methods}

Patients were ascertained prospectively from the private practice of two board certified gynecologic oncologists. All new patients seen over thirty-six months were approached. Patients were a representative sample of lower and middle economic strata and were drawn from both Caucasian and non-Caucasian races. Patients who agreed to participate were mailed a consent form, and upon receipt of the signed consent, a telephone interview was held and their banked tissue was studied. The study was performed in compliance with HIPAA guidelines and was approved by the Institutional Review Board at Saint Barnabas Medical Center, Livingston, NJ. Clinical material primarily reflected a suburban community of middle economic level. Normal tissue contained within the surgical specimens was used as control tissue for all analyses. A body mass index (BMI) of 30 was used as a cutoff for obesity based upon World Health Organization criteria. All histological slides stained with $H \& E$ were examined by one surgical pathologist and the areas for study were identified. Consecutive unstained tissue slides from the blocks were prepared and the corresponding areas were isolated manually under a dissecting microscope. Micro-dissections for neoplastic areas contained at least $75 \%$ tumor cells, and control tissues contained only normal cells. DNA extraction and preparation followed standard protocol, as previously published [8].

\section{Laboratory methods}

The genes selected for analysis were among those previously reported to be altered in endometrial cancers. The regions of the genes that were amplified and the primers used are shown in Supplement 1. All PCR re- actions were performed as previously described [8]. All primer sets for PCR for loss of heterozygosity (LOH) and $\mathrm{MSI}$ had a 5'fluorescent label on the sense strand and a 5'GTGTCTT tail on the anti-sense strand [8]. LOH of the $A P C$ gene was determined through the PCR amplification of the CA repeat microsatellite markers D5S346, D5S1965 and/or D5S492. LOH of the PTEN gene was determined through the PCR amplification of the CA repeat microsatellite markers D10S2491, D10S541 and/or D10S579. To determine $\mathrm{LOH}$, the ratio of the allele band intensities (peak heights) of the neoplastic tissue was divided by the corresponding ratio of the normal mucosa. $\mathrm{LOH}$ was defined as a resultant ratio of less than or equal to 0.5. Point mutation analyses utilized Sanger sequencing.

MSI was detected using the Bethesda panel of markers, with two mononucleotide markers BAT25 and BAT26, and three dinucleotide markers D2S123, D5S346, and D17S250. MSI for a given primer set is defined as a change in the allele pattern, with the appearance of one or more new PCR products relative to those produced by the normal DNA. A tumor is defined as MSI-high if two or more of the five markers had a changed allele pattern, and is referred to as 'MSI'.

The methylation status of the mismatch repair system was ascertained using the SALSA ${ }^{\circ}$ MS-MLPA ${ }^{\circ}$ Methylation-specific DNA detection Kit \#ME011 (MRC-Holland, Amsterdam, The Netherlands) [8]. This kit uses 22 probes containing a Hha1 recognition site to detect the aberrant methylation in seven MMR genes including 5 MLH1 probes, $3 \mathrm{MSH} 2$ probes, $3 \mathrm{MSH} 6$ probes, $1 \mathrm{MSH} 3$ probes, 1 MLH3 probe, 3 PMS2 probes and 6 MGMT probes. Genomic DNA was hybridized overnight with the probe mix. This hybridization mixture was then split for two separate reactions, a ligation reaction, and a dual ligation and Hha1 restriction enzyme cutting reaction. Electrophoresis of PCR fragments was performed on an ABI 3130 Genetic Analyzer with POP7 polymer at $60{ }^{\circ} \mathrm{C}$ and raw data was processed using Genemapper v4.0 software. The Genemapper data were subsequently exported and the methylation status was analyzed using Coffalyser v9.4 software (MRC-Holland website, www.mlpa.com). The "Direct Methylation Status" analysis option was chosen to normalize and analyze the MS-MLPA data. Methylation status for the probe target sites in each sample was determined by comparing the PCR products from the normal DNA to that of the tumor. Ratios of tumor to normal peak areas for a given probe that are between 0.7 and 1.0 are assigned as methylated. Ratios of tumor to normal peak areas for a given probe that are $<0.3$ range is assigned unmethylated. Ratios between 0.3 and 0.7 are considered as partially methylated or hemi-methylated.

\section{Statistical methods}

Continuous variables were summarized using the 
median (range) or mean (SD), with differences across groups assessed using the Wilcoxon Rank-Sum test. The association between categorical variables was assessed using the Chi-Squared or Fisher's Exact test as appropriate. The association between clinical and pathological features with genetic change was estimated using a logistic regression approach, with odds ratios and $95 \%$ confidence intervals reported.

\section{Results}

A total of 212 consecutive patients were approached regarding the study. One hundred five $(49.5 \%)$ agreed to participate, returned the consent form, and completed the telephone interview. One hundred seven patients were non-participants: 49 (23.1\%) failed to return a signed consent form, $32(15.1 \%)$ failed to respond to telephone messages, 17 (8.0\%) declined, 3 (1.4\%) lacked proficiency with English, and $6(2.8 \%)$ had unsuitable or unavailable pathological specimens. There was no difference between the age of the non-participants (64.7 years) and the participants (64.0), and the percentage of cancers that were endometrioid was also the same for the two groups ( $85.7 \%$ for the non-participants and $86.7 \%$ for the participants). Clinical characteristics of the participants are shown in Table 1. These findings are similar to our previous cohort of patients from 1995 to 2007 [9]. Among the $30.5 \%$ of patients who were smokers, the median pack-years were 8.0, with a range of 0.8 to 50.0 years. The mean BMI was similar for those experiencing miscarriage (33.0) and those who did not (31.2) $[p=0.245]$.

\section{Life style characteristics}

Among the 32 patients who used cigarettes, 16 (51.6\%) smoked less than or equal to 10 pack-years at the time of diagnosis, and 15 (48.4\%) had smoked greater than 10 pack-years. Duration was unknown for one patient. Of the 56 patients consuming coffee, all but four drank regular coffee, while four used only decaffeinated coffee. Over half the patients consumed an average of two or more cups per day, with a median of 39.5 years of consumption. Non-steroidal anti-inflammatory drug (NSAID) and vitamin use was defined as consumption on most days of the year, for at least one year prior to cancer diagnosis. Sixty-five patients responded definitively to whether their mothers had taken diethylstilbestrol (DES) while carrying them. Three patients' mothers had taken DES, 62 indicated their mothers had not. The others had no information in this regard. The three women who were 'DES babies' all had endometrioid cancers.

\section{Secondary cancers}

Twenty patients (19\%) reported having had a prior cancer. These included: Breast-9, lung-2, bladder-2, skin-2, and one each for colon, melanoma, kidney, pancreas, and fallopian tube. Eighty-five patients indicated they had not had a second cancer. Three patients were diagnosed with a second cancer within 1 to 2 years of
Table 1: Baseline characteristics of 105 patients with uterine cancer.

\begin{tabular}{|c|c|c|}
\hline & N or (Median) & $\%$ or (Range) \\
\hline Age at diagnosis, yrs & $(64)$ & $(39-92)$ \\
\hline Age $<50$ yrs & 8 & 7.6 \\
\hline Age $\geq 50$ yrs & 97 & 92.4 \\
\hline BMI & $(31.4)$ & $(17.7-66.2)$ \\
\hline $\mathrm{BMI}<30$ & 42 & 40 \\
\hline $\mathrm{BMI} \geq 30$ & 63 & 60 \\
\hline Diabetes & 19 & 18.1 \\
\hline Hypertension & 55 & 52.4 \\
\hline Cigarette use & 32 & 30.5 \\
\hline pack-yr & (8) & $(0.8-50)$ \\
\hline \multicolumn{3}{|l|}{ Coffee } \\
\hline Used & 56 & 67.5 \\
\hline Never used & 27 & 32.5 \\
\hline Unknown & 22 & \\
\hline Menarche age & $(12)$ & $(9-15)$ \\
\hline Menopause age & $(52)$ & $(39-61)$ \\
\hline $\begin{array}{l}\text { Patients with prior } \\
\text { pregnancies }\end{array}$ & 80 & 76.2 \\
\hline No. pregnancies & $(2)$ & $(0-6)$ \\
\hline Patients with miscarriages & 35 & 33.3 \\
\hline Birth control pills used & 31 & 29.5 \\
\hline Post-menopausal estrogen & 13 & 12.5 \\
\hline Vitamin use & 66 & 63.5 \\
\hline NSAID ${ }^{\dagger}$ use & 34 & 32.4 \\
\hline
\end{tabular}

*use unknown for one patient

tnonsteroidal anti-inflammatory drug

the diagnosis of uterine cancer, one patient with colon and two with breast cancer. These three patients were older than 60 years at the time of uterine cancer diagnosis.

\section{Histological and molecular results}

Histopathological features of the cancers are shown in Table 2. The distribution of histological subtypes is consistent with previous publications [10]. Twenty-three cancers with associated hyperplasia were all of the endometrioid histological subtype; complex hyperplasia with atypia was present in 16 (69.6\%) cases, simple hyperplasia in $4(17.4 \%)$ cases, complex hyperplasia in 2 $(8.7 \%)$ cases, and atypical hyperplasia in $1(4.3 \%)$ case. Pathological reports did not indicate the FIGO score for 12 cancers, of which 11 were serous and one was carcinosarcoma.

The molecular findings are shown in Table 3. They are mainly consistent with our previous findings and with results reported by others [9,11-13]. However, our results for abnormalities in PTEN gene are slightly lower than previously reported [14]. No mutations were detected for the $K$-Ras gene at codon 61 , nor in the GNAS gene at codon 201 of exon 8 . Only one cancer contained 
a mutation for $B$-Raf at codon 600 exon 15, and this tumor was also MSI and methylated. Twenty-six cancers with MSI were not technically evaluable for $\mathrm{LOH}$ of the $A P C$ or PTEN genes. Six tumors demonstrated $\mathrm{LOH}$ for the $A P C$ gene.

The distribution of the 19 cancers with a $K$-Ras gene mutation was: c.35G > A-7 (36.8\%), c.38G > A-6 (31.6\%), c35G > C-3 (15.8\%), c.35G > T-2 (10.5\%), and c.34G > T-1 (5.3\%). Twenty-two of the 26 cancers with MSI demonstrated methylation, with four having no methylation. The types of PTEN mutations were: Deletions $=10(47.6 \%)$, insertions $=3(14.3 \%)$, point mutations $=8(38.1 \%)$. Three cancers with a PTEN mutation also demonstrated PTEN LOH.

Table 2: Pathological features of 105 uterine cancers.

\begin{tabular}{|l|l|l|}
\hline & No. & $(\%)$ \\
\hline Cancer subtype & & $(86.7)$ \\
\hline Serous & 91 & $(10.5)$ \\
\hline Carcinosarcoma & 11 & $(1.9)$ \\
\hline Clear cell & 2 & $(1)$ \\
\hline FIGO* & 1 & \\
\hline 1 & & $(39.8)$ \\
\hline 2 & 37 & $(39.8)$ \\
\hline 3 & 37 & $(20.4)$ \\
\hline Hyperplasia & 19 & $(21.9)$ \\
\hline No hyperplasia & 23 & $(78.1)$ \\
\hline TNM stage & 82 & \\
\hline 0 & 1 & $(1)$ \\
\hline 1 & 85 & $(81)$ \\
\hline 2 & 8 & $(7.6)$ \\
\hline 3 & 10 & $(1) 5)$ \\
\hline 4 & 1 & \\
\hline
\end{tabular}

"No score reported for the serous and carcinosarcoma tumors.

\section{Family history}

Family histories are summarized in Table 4. Three of our four patients with uterine cancers demonstrating MSI but no methylation all had strong family histories of cancers in their first-degree relatives. The first patient's sister had ovarian cancer; the second patient's mother had both uterine and breast cancer; the third patient's mother had breast and colon cancer. These three also had numerous second-degree relatives with cancers of ovary, breast, uterus and colon. The fourth patient's two maternal aunts both had colon cancer.

Table 3: Molecular findings of 105 uterine cancers.

\begin{tabular}{|c|c|c|}
\hline & No. & $(\%)$ \\
\hline \multicolumn{3}{|l|}{ KRAS 12/13 } \\
\hline Mutated & 19 & $(18.1)$ \\
\hline Wild & 86 & (81.9) \\
\hline \multicolumn{3}{|l|}{ Microsatellite } \\
\hline Unstable-high & 26 & $(24.8)$ \\
\hline Stable & 79 & $(75.2)$ \\
\hline \multicolumn{3}{|l|}{ Methylation } \\
\hline Yes & 22 & $(84.6)$ \\
\hline No & 4 & $(15.4)$ \\
\hline \multicolumn{3}{|l|}{ APC $^{*}$} \\
\hline $\mathrm{LOH}^{\dagger}$ & 6 & $(7.6)$ \\
\hline Wild type & 73 & $(92.4)$ \\
\hline \multicolumn{3}{|l|}{ PTEN $^{*}$} \\
\hline $\mathrm{LOH}$ & 30 & $(38.0)$ \\
\hline Wild type & 49 & (62.0) \\
\hline \multicolumn{3}{|l|}{ PTEN } \\
\hline Mutated & 21 & $(20.0)$ \\
\hline Wild type & 84 & $(80.0)$ \\
\hline
\end{tabular}

*26 cancers uninformative because of microsatellite instability. † Loss of Heterozygosity

Molecular findings of 105 uterine cancers.

Table 4: Family history of cancers in patients with uterine cancer.

\begin{tabular}{|c|c|c|c|}
\hline Relationship to Patient & First-degree & Second-degree & Third-degree \\
\hline Median \# relatives/patients & 6 & 16 & 13 \\
\hline \# (\%) with a relative with cancer & $76(72.4)$ & $75(71.4)$ & $18(17.1)$ \\
\hline Median age of relatives with cancer & 62 & 62 & 56.5 \\
\hline \multicolumn{4}{|l|}{ \# $(\%)$ cancers found in relatives } \\
\hline Breast & $27(20.1)$ & $41(22.5)$ & $5(27.8)$ \\
\hline Colorectal & $18(13.4)$ & $28(15.4)$ & $2(11.1)$ \\
\hline Lung & $12(9)$ & $23(12.6)$ & 0 \\
\hline Prostate & $12(9)$ & $8(4.4)$ & 0 \\
\hline Uterus & $10(7.5)$ & $8(4.4)$ & $4(22.2)$ \\
\hline Head and neck & $9(6.7)$ & $8(4.4)$ & 0 \\
\hline Gastric & $6(4.5)$ & $12(6.6)$ & $1(5.6)$ \\
\hline $\begin{array}{l}\text { Bladder, brain, cervix, esophagus, Leukemia, lymphoma, kidney, } \\
\text { ovary, pancreas, melanoma, bone }\end{array}$ & each tumor $\leq 5$ & each tumor $\leq 5$ & each tumor $\leq 5$ \\
\hline
\end{tabular}




\section{Personal and family history characteristics in rela- tionship to molecular genetic changes}

Data relating the personal and family histories of the patients to their tumors' molecular genetic changes are summarized in Figure $1 \mathrm{a}$ and Figure $1 \mathrm{~b}$. Age at diagnosis, and age either less than 50 years or equal/greater than 50 years, was significantly related only to the presence of methylation. The median age of 22 tumors with methylation was 66.5 years and was 51.0 years for the 4 tumors without methylation $(p=0.008)$. Twenty-one of the 22 patients with tumors with methylation were over 50 years of age; and only one was less than 50 years at diagnosis. For the entire cohort, we found no relationship between a family history of a first degree relative or a first and/or second degree relative with uterine cancer and any of the molecular changes evaluated.
A body mass index (BMI) under 30 was significantly associated with PTEN mutation, with 13 of 21 tumors demonstrating this mutation found in patients with the lower BMI. We found no relationship between BMI and methylation. Of our 22 patients with methylated tumors, 11 of $14(78.6 \%)$ had a high BMI, while 11 of 12 (91.7\%) had a low BMI (OR $=0.3, \mathrm{Cl}=0.0,3.1, \mathrm{p}=0.598)$. We did not find any significant correlation between the presence of diabetes or hypertension and the various genetic changes. Of note, among the 22 patients with tumors demonstrating MSI and methylation, 18 were non-smokers and only 4 had been smokers. The consumption of coffee did not appear to have any relationship with the genetic changes studied.

Estrogen use, as reflected by prior pregnancies or oral estrogen use was statistically associated with MSI,
A)

No. with genetic change

No. tested for change

\section{Clinical feature}

Age $<50, \geq 50$

$\mathrm{BMI}<$ or $>1=30$

Diabetes $y / n$

Hypertension $y / n$

Cigarette use $y / n$

Coffee use $y / n$

Pregnancies $\mathrm{y} / \mathrm{n}$

Birth control $y / n$

Estrogen use $y / n$

Vitamins $y / n$

Nsaids $y / n$

First-degree relative

with uterine cancer

B)

No. with genetic change

No. tested for change

\section{Clinical feature}

Age $<50, \geq 50$

$\mathrm{BMI}<$ or $>$ / $=30$

Diabetes $\mathrm{y} / \mathrm{n}$

Hypertension $y / n$

Cigarette use $y / n$

Coffee use $y / n$

Pregnancies $\mathrm{y} / \mathrm{n}$

Birth control $y / n$

Estrogen use $y / n$

Vitamins $y / n$

Nsaids $y / n$

First-degree relative with uterine cancer

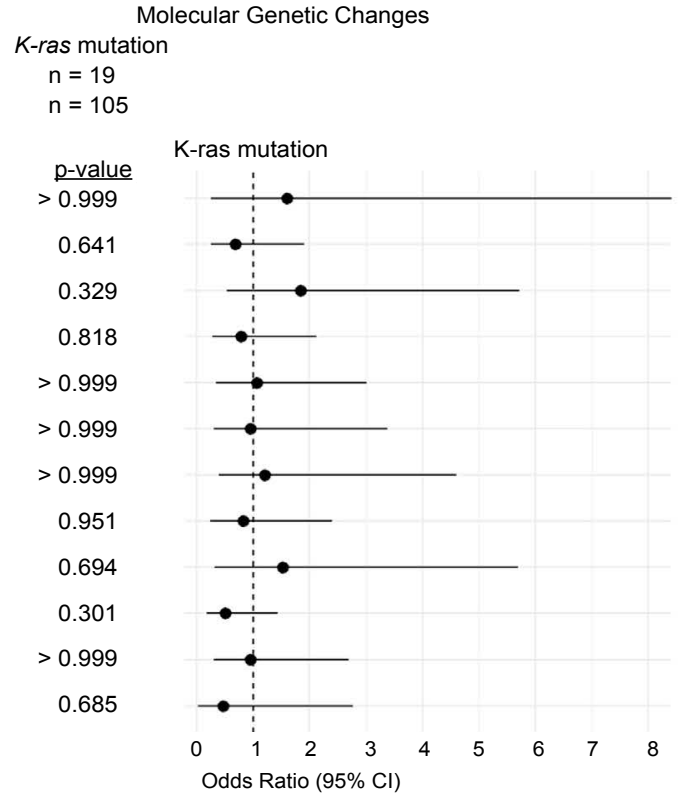

MS

$\mathrm{n}=26$

$\mathrm{n}=105$

p-value MS

0.406

0.612

0.777

0.690

0.093

$>0.999$

0.050

0.930

0.003

0.638

0.315

0.445

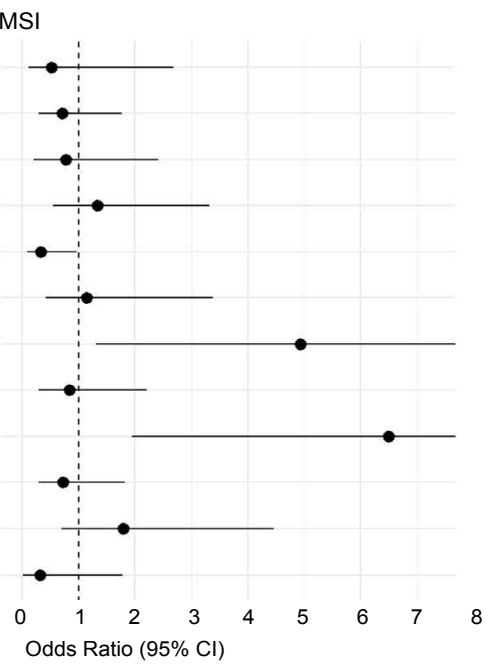

PTEN LOH

$\mathrm{n}=30$

$\mathrm{n}=81$

$\mathrm{n}=21$
$\mathrm{n}=105$

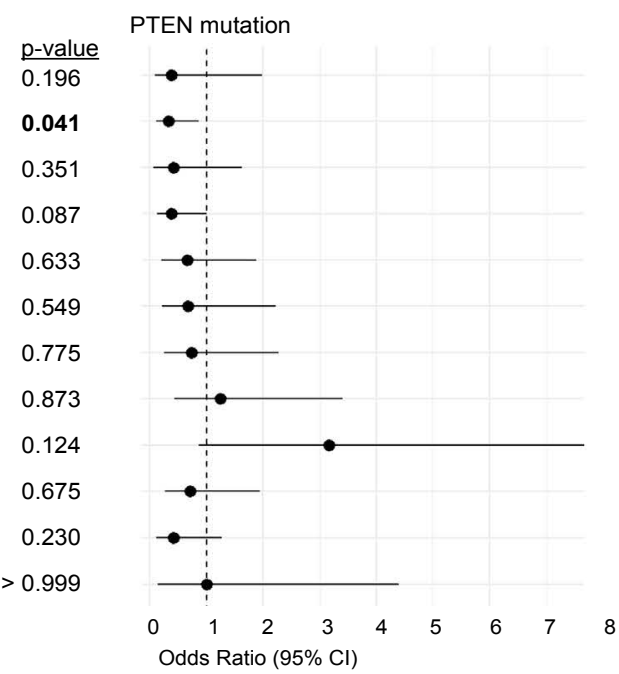

p-value

$>0.999$

0.670

$>0.999$

0.284

0.949

0.926

0.696

0.416

0.644

$\mathbf{0 . 0 2 5}$

$>0.999$

0.724

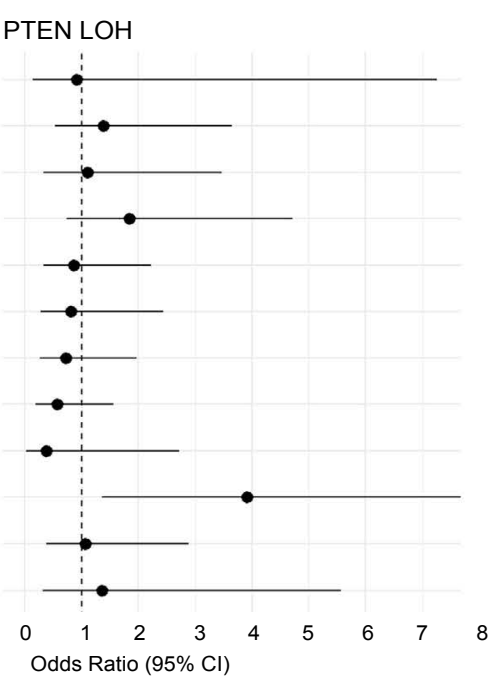

Figure 1: a,b) Clinical features of patients with uterine cancer in relationship to the molecular genetic changes in their cancers. 
but not with the other genetic changes. Twenty-four of $26(92.3 \%)$ tumors with MSI were from patients who had prior pregnancies, while 56 of 79 (70.9\%) of patients with microsatellite stable tumors had prior pregnancies $(p=0.05)$. Similarly, estrogen had been used by 8 of $26(30.8 \%)$ patients with MSI tumors, and just 5 of $78(6.4 \%)$ patients with microsatellite stable tumors ( $p$ $=0.003$ ). We found no relationship between MSI and whether or not the patients had used oral contraceptives; seven of $31(22.6 \%)$ users had tumors with MSI, and 19 of 74 (25.7\%) non-users had tumors with MSI ( $\mathrm{OR}=0.8, \mathrm{Cl}=0.3,2.2, \mathrm{p}=0.930$ ).

The consumption of coffee did not correlate with any of the genetic markers, nor did the use of nonsteroidal anti-inflammatory drugs. The use of vitamins was much more frequent among women with tumors demonstrating PTEN LOH (24 of $29,82.8 \%$ ) compared to women with tumors without PTEN LOH (27 of 49,55.1\%), ( $p=$ 0.025 ). However, vitamin use was equal among women whose tumors contained a PTEN mutation (12 of $21,57.1 \%$ ) and those whose tumors did not (54 of 83 , $65.1 \%)(p=0.675)$. (Figure 1a and Figure $1 b)$.

\section{Discussion}

Most endometrial carcinomas are hormone-dependent tumors and are associated with high circulating levels of unopposed estrogen [15]. Other risk factors include early age of menarche, longer days of menstrual flow, and obesity and diabetes [16]. The risk from obesity may reflect estrogen level, at least for postmenopausal women [17]. Other risk factors include older age, late menopause, a family history of endometrial cancer, radiation exposure, and infertility, particularly in the presence of polycystic ovarian syndrome. Smoking is protective, as a result of its anti-estrogenic effect [18]. Endometrial carcinomas at menopause and beyond may reflect estrogen converted from adrenal androgen production [2]. Many of these risk factors were observed in the 1980s and summarized by Schottenfeld [19].

The cumulative risk of miscarriage for weeks 5-20 of gestation has been reported as $11-22 \%$ [20]. The overall incidence might be higher with respect to a longer gestational period. One-third of our patients reported having experienced a miscarriage, suggesting that perhaps the overall incidence for our patients was within the range of the general population. However, our finding that one-third of the patients had used cigarettes is above the New Jersey average of $14 \%$.

A recent review suggested the prevalence of Lynch syndrome in endometrial cancer patients is approximately 3\% [21]. Our four cancers with MSI and no methylation are consistent with Lynch syndrome, or HNPCC, as is the family history of these four patients. Overall, family history of uterine cancer in a first degree and/or second degree relative was not related to any of the genetic changes we evaluated. This suggests that certain germ line genetic changes may predispose a woman to the development of uterine cancer, but the specific changes within the tumor may develop from multiple other influences. The literature related to age and MSI is contradictory. We found no relationship between age and MSI, whether analyzed as a continuous or as a categorical variable. The median age for patients with microsatellite stable tumors was 64.0 years and it was 65.0 years for those with MSI tumors ( $p=0.727)$. We did, however, find methylation to be related to older age of the patient.

One study demonstrated that oral contraceptive use is associated with a reduced risk for microsatellite unstable endometrial cancer [22]. We found the use of oral contraceptives had no effect on microsatellite instability, as assessed with the markers we used. It is possible that our patients, in general, used oral contraceptives for only short periods, insufficient to affect uterine carcinogenesis. In addition, the combining of progestin with the estrogen in oral contraceptives may have negated any adverse estrogenic effect. McCourt, et al. reported that women with MSI positive tumors had a lower BMI than those women with microsatellite stable tumors [23]. Our data indicated no relationship between BMI and microsatellite status of the tumors. BMI was reported to be associated with DNA methylation in carcinoma of the endometrium [24]. One proposed mechanism to explain this relationship suggests that obesity promotes adipose inflammation and increased estrogen signaling in adipose depots [25]. Our results do not support this association.

We did find several other correlations between the patients' clinical findings and the genetic changes in their tumors. Both pregnancy and use of estrogen were strongly correlated with the presence of microsatellite instability, suggesting this is a true relationship. Smoking was not a risk for the presence of microsatellite instability. A lower BMI was associated with almost a twofold greater likelihood of the patients' tumors having a PTEN mutation, suggesting that obesity $(\mathrm{BMI}>30)$ is 'protective' against developing this mutation. Alternatively, it may be that obesity, with the various associated metabolic changes, leads to other molecular genetic changes which drive the neoplastic process.

We found a correlation between multi-vitamin use and PTEN LOH. Although vitamins and their metabolites have been reported to effect gene regulation and expression, it is not clear what the exact mechanism for this relationship might be [26]. Finally, we found PTEN mutations to be more frequent than PTEN wild-type in tumors with MSI, perhaps as a consequence of the generalized genetic instability. However, a K-ras mutation was not more frequent in tumors with MSI than was K-ras wild-type.

There are several limitations to this study. Since no single molecular genetic change is necessarily present 
in all uterine tumors, some of the subgroups are limited in number. Multiple group testing may inadvertently result in the finding of a false positive relationship. We employed focused molecular testing. Furthermore, our data were not suitable to apply Mendelian randomization. The challenge for the future is to combine large studies of molecular genetics and pathologic data with consistent clinical information about the patients. Only with robust data will it be possible to fully understand the possible effects of exogenous influences on the various genetic changes in cancers.

\section{Acknowledgements}

The authors thank Dr. Errol Berman (deceased) for review of histological slides.

\section{Competing Interests}

The authors declare no competing interests.

\section{Funding}

The Harvey Nussbaum Foundation of Saint Barnabas Medical Center and the June Bleiwise Foundation.

\section{Sources of Support}

The Harvey Nussbaum Foundation of Saint Barnabas Medical Center and the June Bleiwise Foundation.

\section{Authors Contribution}

All authors have provided substantial contribution and they are all in agreement with all aspects of the final manuscript.

\section{References}

1. Bokhman JV (1983) Two pathogenetic types of endometrial carcinoma. Gynecol Oncol 15: 10-17.

2. Sivridis E, Giatromanolaki A (2011) Pathogenesis of endometrial carcinomas at menopause: Facts and figures. J Clin Pathol 64: 553-560.

3. Cancer Genome Atlas Research Network, Kandoth C, Schultz N, Cherniack AD, Akbani R, et al. (2013) Integrated genomic characterization of endometrial carcinoma. Nature 497: 67-73.

4. Azueta A, Gatius S, Matias-Guiu X (2010) Endometrioid carcinoma of the endometrium: Pathologic and molecular features. Semin Diagn Pathol 27: 226-240.

5. Hecht JL, Mutter GL (2006) Molecular and pathologic aspects of endometrial carcinogenesis. J Clin Oncol 24: 47834791.

6. Kuismanen SA, Moisio AL, Schweizer P, Truninger K, Salovaara R, et al. (2002) Endometrial and colorectal tumors from patients with hereditary nonpolyposis colon cancer display different patterns of microsatellite instability. Am J Pathol 160: 1953-1958.

7. Slattery ML, Curtin K, Anderson K, Ma KN, Ballard L, et al. (2000) Associations between cigarette smoking, lifestyle factors and microsatellite instability in colon tumors. J Natl Cancer Inst 92: 1831-1836.

8. Zauber P, Denehy T, Taylor R, Ongcapin E, Marotta S, et al. (2015) Strong correlation between molecular changes in endometrial carcinomas and concomitant hyperplasia. Int $\mathrm{J}$ Gynecol Cancer 25: 863-868.

9. Zauber NP, Denehy TR, Taylor RR, Ongcapin E, Marotta SP, et al. (2010) Microsatellite instability and DNA methylation of endometrial tumors and clinical features in young women compared with older women. Int J Gynecol Cancer 20: 1549-1556.

10. Bell DW, Ellenson LH (2019) Molecular genetics of endometrial carcinoma. Annu Rev Pathol 14: 339-367.

11. Dong F, Costigan DC, Howitt BE (2019) Targeted next-generation sequencing in the detection of mismatch repair deficiency in endometrial cancers. Mod Pathol 32: 2527.

12. Cornel KMC, Wouters K, van de Vijver KK, Van der Wurff AAM, Engeland M, et al. (2019) Gene promoter methylation in endometrial carcinogenesis. Pathol Oncol Res 25: 659-667.

13. Haruma T, Nagasaka T, Nakamura K, Haraga J, Nyuya A, et al. (2018) Clinical impact of endometrial cancer stratified by genetic mutational profiles, POLE mutation, and microsatellite instability. PloS One 13: e0195655.

14. Soumerai TE, Donoghue MTA, Bandlamudi C, Srinivasan $\mathrm{P}$, Chang MT, et al. (2018) Clinical utility of prospective molecular characterization in advanced endometrial cancer. Clin Cancer Res 24: 5939-5947.

15. I Persson, HO Adami, L Bergkvist, A Lindgren, B Pettersson, et al. (1989) Risk of endometrial cancer after treatment with oestrogens alone or in conjunction with progestogens: Results of a prospective study. Br Med J 298: 147-151.

16. Brinton LA, Berman ML, Mortel R, Twiggs LB, Barrett RJ, et al. (1992) Reproductive, menstrual, and medical risk factors for endometrial cancer: Results from a case-control study. Am J Obstet Gynecol 167: 1317-1325.

17. Zeleniuch-Jacquotte A, Akhmedkhanov A, Kato I, Koenig $\mathrm{KL}$, Shore RE, et al. (2001) Postmenopausal endogenous estrogens and risk of endometrial cancer: Results of a prospective study. Br J Cancer 84: 975-981.

18. Baron JA, La Vecchia C, Levi F (1990) The antiestrogenic effect of cigarette smoking in women. Am J Obstet Gynecol 162: 502-514.

19. Schottenfeld D, Fraumeni JE (2008) Cancer epidemiology and prevention. ( $3^{\text {rd }}$ edn), New York, NY: Oxford University Press 1027-1043.

20. Ammon-Avalos L, Galindo C, Li DK (2012) A systematic review to calculate background miscarriage rates using life table analysis. Birth Defects Res 94: 417-423.

21. Ryan NAJ, Glaire MA, Blake D, Cabrera-Dandy M, Evans DG, et al. (2019) The proportion of endometrial cancers associated with Lynch syndrome: A systematic review of the literature and meta-analysis. Genet Med 21: 2167-2180.

22. Amankwah EK, Friedenreich CM, Magliocco AM, Brant R, Speidel T, et al. (2013) Hormonal and reproductive risk factors for sporadic microsatellite stable and unstable endometrial tumors. Cancer Epidemiol Biomarkers Prev 22: 1325-1331.

23. McCourt CK, Mutch DG, Gibb RK, Rader JS, Goodfellow PJ, et al. (2007) Body mass index: Relationship to clinical, pathologic and features of microsatellite instability in endometrial cancer. Gynecol Oncol 104: 535-539.

24. Gu Y, Zhang CW, Wang L, Zhao Y, Wang Hui, et al. (2018) Association analysis between body mass index and genomic DNA methylation across 15 major cancer types. $J$ Cancer 9: 2532-2542.

25. Howe LR, Subbaramaiah K, Hudis CA, Dannenberg AJ 
(2013) Molecular pathways: Adipose inflammation as a mediator of obesity-associated cancer. Clin Cancer Res 19: 6074-6083.
26. Van der Knapp, Verrijzer CP (2016) Undercover: Gene control by metabolites and metabolic enzymes. Genes Dev 30: 2345-2369.

Supplement 1: Primers used for analyses of gene mutations.

\begin{tabular}{|c|c|c|c|c|}
\hline Gene sequence & Codon & Exon & Primer set $^{*}$ & Reaction sequence \\
\hline \multirow[t]{2}{*}{ K-ras } & $12 / 13$ & 2 & 5'-AAGGCCTGCTGAAAATGACTG-3' & \multirow[b]{2}{*}{ reverse } \\
\hline & & & 5'-GGTCCTGCACCAGTAATATGCA-3' & \\
\hline \multirow[t]{2}{*}{ K-ras } & 61 & 3 & 5'-CAGACTGTGTTTCTCCСTTCTC-3' & \multirow[b]{2}{*}{ forward } \\
\hline & & & 5'-СССТCСССАGTССТСATG-3' & \\
\hline \multirow[t]{2}{*}{$B R A F$} & 600 & 15 & 5'-CATAATGCTTGCTCTGATAGGAAA-3' & \multirow[b]{2}{*}{ forward } \\
\hline & & & 5'-GATCCAGACAACTGTTCAAACTG-3' & \\
\hline \multirow[t]{2}{*}{ GNAS } & 201 & 8 & 5'-ACTGTTTCGGTTGGCTTTGGTGA-3' & \multirow[b]{2}{*}{ reverse } \\
\hline & & & 5'AGGGACTGGGGTGAATGTCAAGA-3' & \\
\hline \multirow[t]{2}{*}{ PTEN } & 7 & & 5'-TGACAGTTTGACAGTTAAAGG-3' & \multirow[b]{2}{*}{ forward } \\
\hline & & & 5'-GGATATTTCTCCCAATGAAAG -3' & \\
\hline \multirow[t]{4}{*}{ PTEN } & 8 & & 5'-TTAAATATGTCATTTCATTTCTTTT-3'/ & \multirow[b]{4}{*}{ reverse } \\
\hline & & & 5'TTGCTTTGTCAAGATCATT-3' & \\
\hline & & & 5'-GTGCAGATAATGACAAGGAATA-3'/ & \\
\hline & & & 5'-TCATGTTACTGCTACGTAAAC-3' & \\
\hline
\end{tabular}

*Obtained from Sigma Life Science oligomer service (http://www.sigmaaldrich.com/life-science/custom-oligos/custom-dna.html)

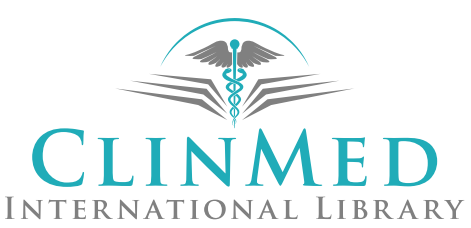

